\title{
Análise comparativa da resistência à fadiga por fretting entre os cabos condutores feitos de liga de alumínio 900 MCM e 823 MCM
}

\author{
Comparative analysis of fretting fatigue resistance \\ between overhead conductors made of aluminum \\ alloys $900 \mathrm{MCM}$ and $823 \mathrm{MCM}$
}

\author{
Erick Rocha Vieira ${ }^{1}$, Jorge Luiz de Almeida Ferreira ${ }^{1}$, \\ José Alexander Araújo ${ }^{1}$, Remy Badibanga Kalombo ${ }^{1}$, \\ Cosme Roberto Moreira da Silva ${ }^{1}$
}

\begin{abstract}
${ }^{1}$ Grupo de Fadiga, Fratura e Materiais, GFFM/ENM/UnB, Universidade de Brasília, Laboratório de Engenharia Mecânica, SG 09, Asa Norte, CEP: 70910-900, Brasília, Distrito Federal, Brasil

e-mail: rochaverick@gmail.com,jorgelaf2012@gmail.com, jaaunb@gmail.com, badibanga12@gmail.com, cosme.roberto@gmail.com
\end{abstract}

\begin{abstract}
RESUMO
Com a necessidade de aperfeiçoamento do processo de transmissão e distribuição de energia elétrica, as indústrias e concessionárias desse setor estão em busca de componentes mais otimizados para suas linhas de transmissão. Em consequência, há a necessidade de aprimorar a formação do cabo condutor, que é o elemento mais oneroso das linhas elétricas. Nestas circunstâncias, condutores formados apenas por fios de alumínio aparentam ser mais vantajosos em relação aos cabos com alma de aço, obtendo melhores índices de ampacidade/peso, diminuindo assim, o custo de transmissão. Neste cenário, o objetivo deste artigo, foi verificar a influência do fenômeno de fadiga por fretting sob condições controladas, nos fios de liga de alumínio AA 6201 T81 e AA 1120 H19, que formam os condutores CAL 900 MCM e CAL 823 MCM, respectivamente. Para alcançar tal fim, criou-se um programa experimental capaz de adquirir os dados sobre a resistência à fadiga dos cabos condutores e dos fios de forma separada, a fim de analisar a influência do fretting sob o parâmetro de carga normal de contato de $750 \mathrm{~N}$. Ao examinar os resultados, o condutor CAL 900 MCM obteve a menor resistência à fadiga, apesar de ser constituído por uma liga com melhores propriedades mecânicas. $\mathrm{O}$ mesmo resultado foi obtido nos ensaios de fadiga por fretting nos fios, deste modo, pôde-se inferir que a liga AA 6201 T81, embora possua melhor resistência à fadiga, ela é mais suscetível a falha na presença de fretting quando comparada a liga AA 1120 H19.
\end{abstract}

Palavras chaves: fadiga por fretting em fios, contato entre fios de alumínio liga AA 1120 H19, liga AA 6201 T81.

\section{ABSTRACT}

The electric transmission and distribution industries and utilities are looking for the most optimized components for their transmission lines. Thus, there is a need to improve the formation of the overhead conductor, which is the costliest element of electric transmission lines. Under these circumstances, conductors formed solely of aluminum wires appear to be more advantageous over aluminum conductor steel reinforced, obtaining better ampacity/weight ratios, thus lowering the transmission cost. The objective of this article was to verify the influence of the fretting fatigue phenomenon under controlled conditions, on the AA 6201 T81 and AA $1120 \mathrm{H} 19$ aluminum alloy wires, which form the overhead conductors CAL 900 MCM and CAL 823 MCM, respectively. To achieve these goals, an experimental program was created capable of acquiring the data on the fatigue life of the overhead conductors and the wires separately, in order to verify the influence of 
fretting under a $750 \mathrm{~N}$ normal contact load parameter. In the results, the CAL $900 \mathrm{MCM}$ achieved the lowest fatigue life, despite it made of an alloy with better mechanical properties. The same result was obtained in the fretting fatigue tests on wires, so it can be inferred that the AA 6201 T81 alloy, although it has better fatigue resistance, is more susceptible to failure in the presence of fretting when compared to AA $1120 \mathrm{H} 19$ alloy.

Keywords: fretting fatigue on wires, contact between aluminum wires, AA 1120 H19 alloy, AA 6201 T81 alloy.

\section{INTRODUÇÃO}

Segundo o Northeast Group [1] estão previstos gastos globais de U\$ 3,2 trilhões até 2026 em distribuição e transmissão de energia elétrica por partes das concessionárias do setor elétrico. No âmbito nacional, segundo o Operador Nacional do Sistema Elétrico (ONS) [2], o Brasil possui atualmente $141.388 \mathrm{~km}$ de linhas de transmissão de alta tensão variando de $230 \mathrm{kV}$ a $800 \mathrm{kV}$, e está em plena expansão para alcançar $185.484 \mathrm{~km}$ até 2023. Por consequência, nota-se a grande necessidade de sistematização de pesquisas científicas relacionadas a transmissão de eletricidade.

De acordo com o Conselho Internacional de Grandes Redes Elétricas (CIGRE) [3] o cabo condutor é o elemento mais vital para o devido funcionamento da transmissibilidade elétrica, tendo um custo associado de até $40 \%$ do orçamento total das linhas de transmissão. Quando o cabo está em operação ele sofre com vibrações advindas de ações eólicas, esse fenômeno é resultante da formação regular de vórtices devido a iteração entre o cabo e o ar. Nas regiões onde há a restrição do movimento do condutor, tais como: espaçadores, emendas, esferas sinalizadores, amortecedores e principalmente grampos de suspensão, existe a geração de flexão alternada, que geram inúmeros problemas mecânicos que potencializam a falha do condutor nessas regiões $[4,5]$.

Existem vários tipos de falhas mecânicas inerentes ao condutor, tais como: desgaste, abrasão, fadiga, etc. Diversos pesquisadores capacitados nessa área, mostram em seus trabalhos [6-11] que o processo de falha relacionado a fadiga que ocorre no cabo condutor é denominado no meio acadêmico de fadiga por fretting. $\mathrm{O}$ fenômeno de fadiga por fretting ocorre nos condutores devido ao escorregamento parcial existente entre os fios que estão em contato, próximo aos pontos de restrição de movimento. Nessas regiões, há presença de forças normais e tangenciais aliadas a fricção dos fios, causando assim o fretting. Uma vez que a trinca é iniciada pela indução do fretting, poderá propagar-se por fadiga devido ao movimento oscilatório do cabo, podendo levar a falha parcial ou total do condutor [12-14].

Existem inúmeros tipos de condutores utilizados nas linhas de transmissão como: Condutor de Alumínio Puro (CA), Condutor de Alumínio Liga (CAL), Condutor de Alumínio com Alma de Aço (CAA) e Condutor de Alumínio com Alma de Liga de Alumínio (ACAR). A grande maioria dos cabos utilizados no Brasil são do tipo CAA, principalmente os que são formados por AA $1350 \mathrm{H} 19$, que possui boa condutibilidade elétrica, porém com baixa resistência mecânica em relação a outras ligas como AA 6201 T81 e AA 1120 H19. Devido a essa propriedade, os cabos formados por essa liga precisam adotar alma de aço em sua composição para agregar melhor resistência mecânica, que por consequência, torna a linha de transmissão mais pesada, sendo necessária a construção de torres de sustentação mais robustas e menos espaçadas, acarretando aumento nos custos de transmissão.

Os condutores do tipo CAL surgem como alternativa mais econômica por não necessitarem de alma de aço. Contudo, ainda são necessárias maiores elaborações de estudos sistematizados sobre tal assunto. Neste contexto, este artigo utilizou-se dois condutores do tipo CAL: o 900 MCM, formado pela liga AA 6201 T81, e o $823 \mathrm{MCM}$, formado pela liga AA $1120 \mathrm{H} 19$. Com o objetivo de realizar um programa experimental que permita a comparação de vida em fadiga desses dois condutores, além de isolar o fenômeno de fretting sob condições controladas nos fios constituídos pelas ligas AA 6201 T81 e AA 1120 H19, possibilitando de tal modo a compreensão mais efetiva sobre o efeito do contato entre os fios que formam tais condutores elétricos.

\subsection{Fundamentação teórica}

A explanação das seguintes formulações tem o objetivo de situar leitor sobre teoria que embasa os ensaios de fadiga dos cabos condutores, através da formulação de Poffenberger-Swart e curva Tensão-Vida (Curva S-N). A curva de Wöller, também chamada de curva Tensão-vida (S-N) é uma forma de caracterizar o desempenho dos materiais, onde correlaciona a vida em ciclos de um componente com sua tensão nominal. Sua formulação está descrita pela equação (1). 


$$
\sigma_{a}=A N_{i}^{B}
$$

onde, $\sigma_{a}$ é a amplitude de tensão em $\mathrm{MPa}, \mathrm{N}$ é a vida em quantidade de ciclos, e os parâmetros $\mathrm{A}$ e $\mathrm{B}$ são constantes do material.

Para obter a amplitude de tensão associada ao condutor é necessário utilizar a formulação clássica desenvolvida por Poffenberger-Swart (P-S) [15], para obtenção da tensão mecânica associada ao cabo. Este método é utilizado pelo Instituto de Engenheiros, Eletricistas e Eletrônicos (IEEE) desde 1966 como padrão para a aferição de vibrações nos condutores. Nesta formulação a amplitude do deslocamento é transformada em tensão mecânica através da analogia da montagem condutor/ferragens com a viga de Euler, como mostra a figura 1, deste modo a tensão mecânica é dada pela equação (2).

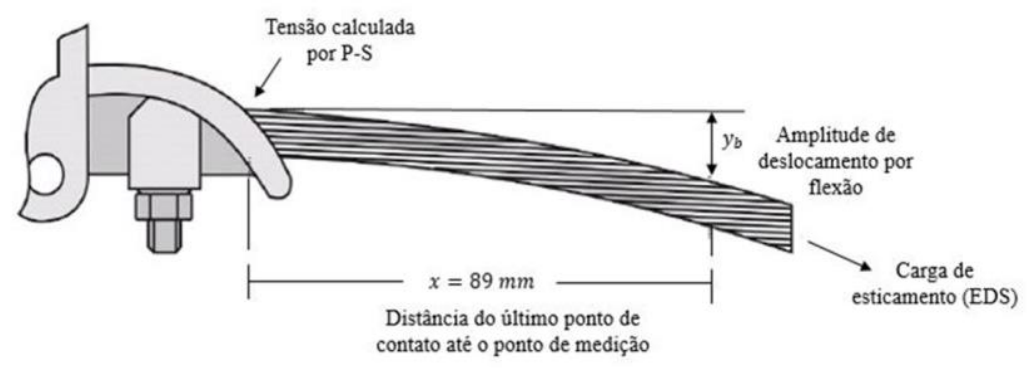

Figura 1: Montagem cabo/grampo

$$
\sigma_{a}=K Y_{b}
$$

onde, $\sigma_{a}[\mathrm{MPa}]$ é a amplitude de tensão dinâmica (zero a pico), $\mathrm{Y}_{b}[\mathrm{~mm}]$ é a amplitude de deslocamento (pico a pico), medida a partir de um ponto posicionado a uma distância $x$ do Último Ponto de Contato (UPC) entre o condutor e a ferragem (adotando-se $x$ igual a $89 \mathrm{~mm}$ ) e $K$ é a constante de Poffenberger, cuja expressão é apresentada na equação (3).

$$
K=\frac{E_{a} d p^{2}}{4\left(e^{-p x}-1+p x\right)}
$$

onde, os parâmetros, $E_{a}[\mathrm{MPa}], d[\mathrm{~mm}]$ e $p[1 / \mathrm{mm}]$ são respectivamente, o módulo de elasticidade (módulo de Young), o diâmetro dos fios de alumínio da camada externa do cabo e a rigidez da montagem, cujo valor é calculado pela equação (4).

$$
p=\sqrt{\frac{T}{E I}}
$$

onde, $T[\mathrm{~N}]$ é a carga de esticamento para a temperatura ambiente, $E I\left[\mathrm{~N} \cdot \mathrm{mm}^{2}\right]$ é a rigidez a flexão do cabo condutor, cujo valor é calculado pela equação (5).

$$
E I_{\min }=\frac{n_{a} E_{a} \pi d_{a}^{4}}{64}+\frac{n_{s} E_{s} \pi d_{s}^{4}}{64}
$$

onde, $n_{a}, E_{a}$ e $d_{a}$ são, o número, o módulo de elasticidade e o diâmetro nominal dos fios de alumínio, respectivamente. E $n_{s}, E_{s}$ e $d_{s}$ são o número, o módulo de elasticidade e o diâmetro nominal dos fios de aço, respectivamente, se caso existir.

\section{MATERIAIS E MÉTODOS}

Para compreender os parâmetros que estão envolvidos no processo de fadiga dos condutores, criou-se um programa experimental dividido em três etapas. A primeira etapa consistiu na caracterização do material, ou seja, na obtenção das propriedades mecânicas (limite de resistência a tração, módulo de elasticidade e resis- 
tência à fadiga) inerentes as ligas formadoras dos cabos condutores. A segunda etapa foi constituída pelos ensaios de fadiga dos condutores na bancada experimental do Laboratório de Fadiga e Integridade Estrutural de Cabos Condutores de Energia da Universidade de Brasília. E finalmente, na terceira parte do experimento, isolou-se o fenômeno de fadiga por fretting nos fios dos condutores através de ensaios controlados, a fim de obter a resistência à fadiga de cada uma das ligas na presença de contato mecânico.

\subsection{Materiais}

Foram usados três cabos condutores nesta pesquisa, sendo eles: CAL 900 MCM, CAL 1055 MCM e CAL 823 MCM. Os dois primeiros condutores são formados por fios da liga AA 6201 T81 e o último pela liga de alumínio AA 1120 H19. Dos cabos formados pela liga AA 6201 T81, o 900 MCM foi utilizado para os ensaios de fadiga dos condutores, e o condutor 1055 MCM como matéria prima dos corpos de prova utilizados nos ensaios dos materiais e de fadiga por fretting. O condutor $823 \mathrm{MCM}$ foi empregado em todos as classes de ensaios mecânicos. A tabela 1 apresenta as características dos condutores utilizados nesta pesquisa.

Tabela 1: Características dos condutores

\begin{tabular}{cccccc}
\hline $\begin{array}{c}\text { CONDUTOR } \\
\text { ELÉTRICO }\end{array}$ & $\begin{array}{c}\text { LIGA DE } \\
\text { ALUMÍNIO }\end{array}$ & $\begin{array}{c}\text { SEÇÃO TRANSVERSAL } \\
\left(\mathbf{M M}^{2}\right)\end{array}$ & $\begin{array}{c}\text { NÚMERO } \\
\text { DE FIOS }\end{array}$ & $\begin{array}{c}\text { DIÂMETRO DOS } \\
\text { FIOS (MM) }\end{array}$ & $\begin{array}{c}\text { CARGA DE RUPTURA } \\
\text { (KN) }\end{array}$ \\
\hline $900 \mathrm{MCM}$ & AA 6201 T81 & 456,00 & 37 & 3,96 & 98,04 \\
\hline $1055 \mathrm{MCM}$ & AA 6201 T81 & 534,46 & 61 & 3,34 & 152,48 \\
\hline $823 \mathrm{MCM}$ & AA 1120 H19 & 417,42 & 37 & 3,79 & 95,17 \\
\hline
\end{tabular}

\subsection{Procedimentos experimentais}

\subsubsection{Ensaios de caracterização dos materiais}

Nesta etapa foram obtidas as propriedades mecânicas das ligas AA 6201 T81 e AA 1120 H19. Na literatura é possível encontrar essas propriedades para a liga AA 6201 T81, como é evidenciado nas referências [16, 17], porém para essa liga da série $1 \mathrm{XXX}$ dados relacionados principalmente a resistência à fadiga são mais escassos na literatura.

Para obter as propriedades mecânicas estáticas, tais como resistência a tração e módulo de elasticidade, foram realizados ensaios monotônicos de tração. Para a obtenção das propriedades dinâmicas, como a resistência à fadiga, foram realizados ensaios cíclicos controlados por força utilizando a metodologia Tensão-Vida (S-N) e a norma ASTM E739-91 (2004) [18], para obter a quantidade necessária de amostras ensaiadas. Para Os parâmetros predefinidos para os ensaios dinâmicos foram: frequência do atuador de $15 \mathrm{~Hz}$; e razão de tensões, $R=0,1$, para impor tensão média na condição de tração-tração, a fim de simular as condições que o cabo condutor experimenta quando está em operação. Para ambas configurações de ensaios, utilizou-se a máquina universal de ensaios servo-hidráulica, MTS Landmark, com célula de carga de $5 \mathrm{kN}$.

Os corpos de prova utilizados foram confeccionados a partir dos fios retirados dos respectivos cabos condutores, e posteriormente foram usinados em um torno $\mathrm{CNC}$, a fim de garantir as proporções estabelecidas pela norma ASTM E466-15 [19]. As dimensões para cada corpo de prova são mostradas na figura 2 e na tabela 2. Seguindo a norma ASTM E739-91 (2004) [18], são necessários pelo menos 6 corpos de prova para obter a taxa de replicação superior a $33 \%$, neste caso, foi utilizado 13 amostras para a liga AA 6201 T81, e 9 amostras para a liga AA 1120 H19, garantindo assim um maior nível de confiabilidade. 


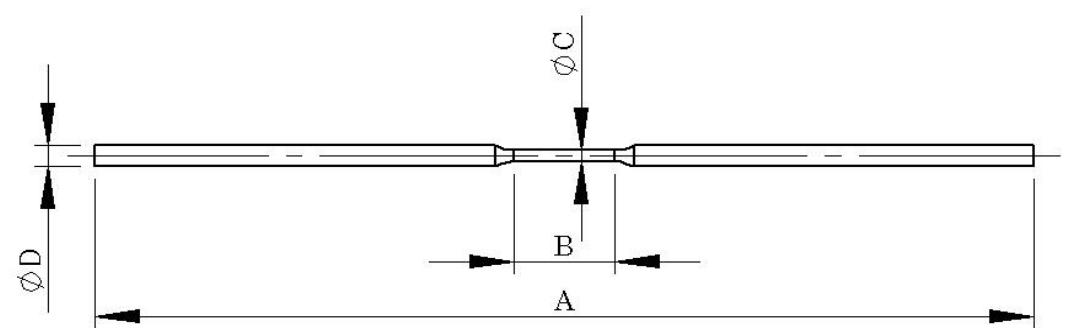

Figura 2: Corpo de prova utilizado nos ensaios de caracterização dos materiais

Tabela 2: Dimensão dos corpos de prova utilizados na caracterização dos materiais

\begin{tabular}{ccccc}
\hline CORPO DE PROVA & A (MM) & B (MM) & C (MM) & D (MM) \\
\hline Estático, AA 6201 T81 & 140,0 & 35,0 & 1,8 & 3,34 \\
\hline Fadiga, AA 6201 T81 & 140,0 & 15,0 & 1,8 & 3,34 \\
\hline Estático, AA 1120 H19 & 140,0 & 35,0 & 2,0 & 3,79 \\
\hline Fadiga, AA 1120 H19 & 140,0 & 15,0 & 2,0 & 3,79 \\
\hline
\end{tabular}

\subsubsection{Ensaios de fadiga dos cabos condutores}

Os condutores CAL 823 MCM e 900 MCM foram ensaiados em condição de fadiga utilizando a bancada de Ensaios Mecânicos à Fadiga de Cabos Condutores de Energia do Laboratório de Fadiga e Integridade Estrutural de Cabos Condutores de Energia, do Departamento de Engenharia Mecânica, da Universidade de Brasília. A ilustração da bancada pode ser encontrada na figura 3.

Inicialmente fixou-se o condutor nos blocos 1 e 3, e em seguida monta-se o grampo de suspensão fixado ao bloco 3. Posteriormente o cabo é esticado com seu respectivo valor de EDS (Every Day Stress). Um excitador eletrodinâmico foi utilizado para simular as vibrações sofridas pelo condutor durante sua operação. Além disso foram utilizados sensores de rotação para verificar a quebra dos fios, estes sensores foram fixados no primeiro nó do condutor em relação ao grampo de suspensão em direção ao excitador eletrodinâmico.

Uma série de ensaios de fadiga foram realizados nos condutores $823 \mathrm{MCM}$ e $900 \mathrm{MCM}$, utilizando carga de esticamento, EDS de $20 \%$ em relação ao limite de resistência a tração do cabo, além disso todos os testes foram realizados seguindo o padrão da IEEE [5] e CIGRE (Conselho Internacional de Grandes Sistemas Elétricos) [20], que definem o critério de falha do condutor ao atingir uma quebra superior a $10 \%$ dos fios. 


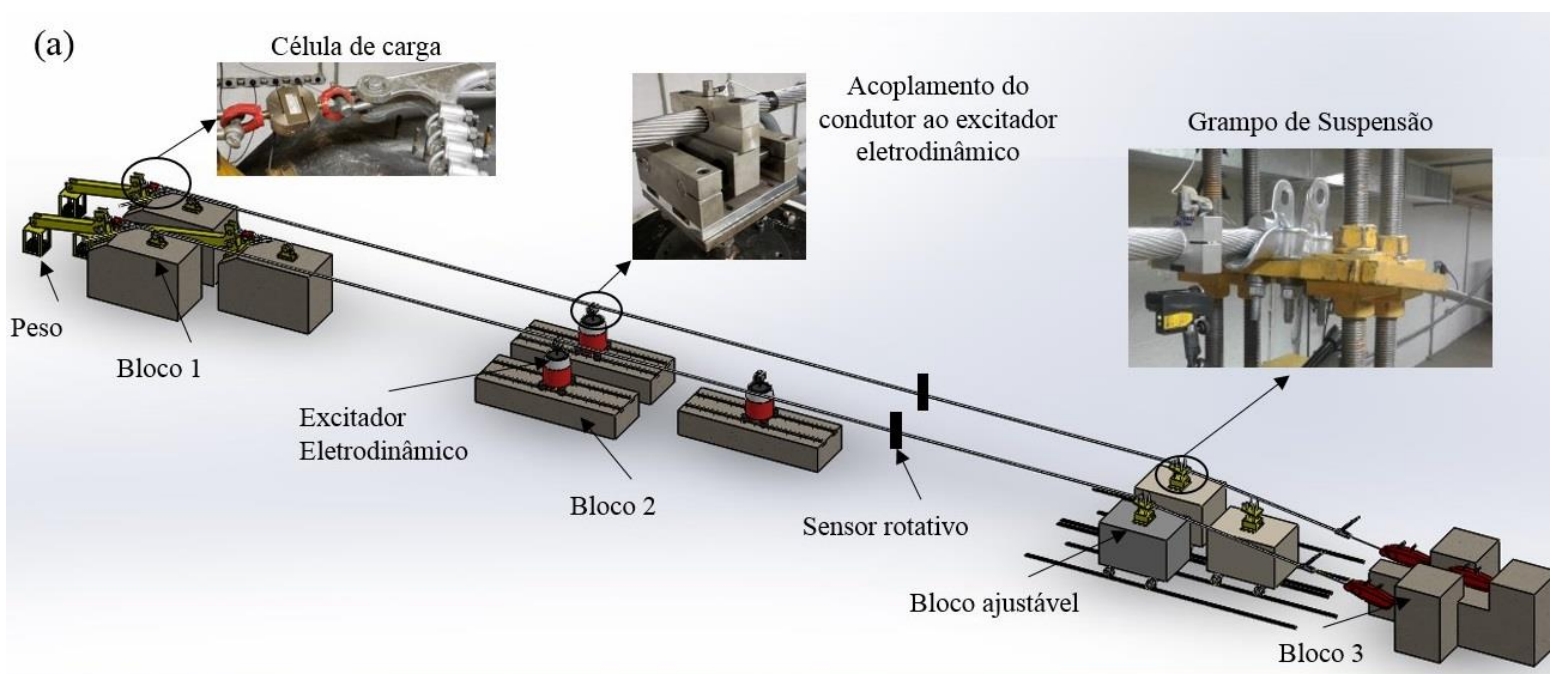

(b)

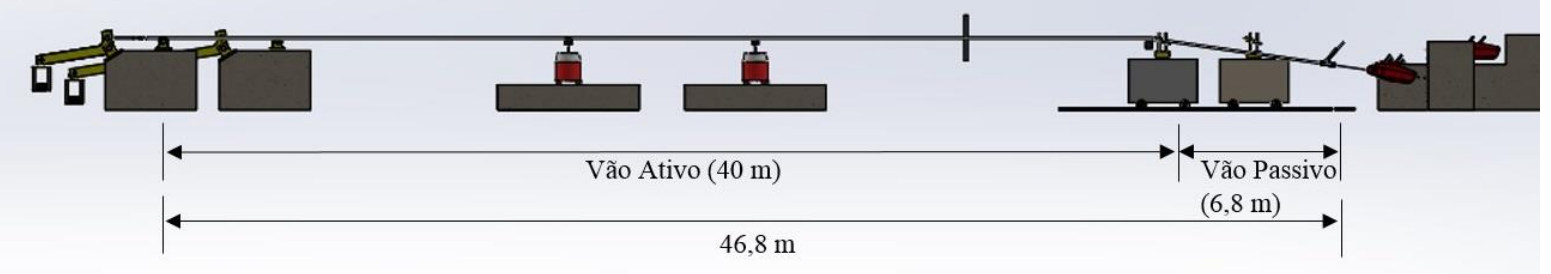

Figura 3: Bancada experimental de ensaios mecânicos

Para medir a amplitude de tensão e consequentemente validar a teoria de Poffenberger-Swart, foram instalados no condutor três extensômetros nos três fios mais acima diametralmente opostos ao UPC. Os parâmetros de temperatura, amplitude de deslocamento pico a pico, $y_{b}$, medido a $89 \mathrm{~mm}$ a partir do UPC e a frequência de ensaio foram controlados em todos os testes de fadiga (ver tabela 3). O vão ativo, região entre as restrições do cabo condutor, foi de 40 metros. Após os ensaios, utilizou-se a metodologia S-N para gerar os gráficos com os dados obtidos nesta etapa.

Tabela 3: Parâmetros dos ensaios de fadiga dos condutores

\begin{tabular}{|c|c|c|c|c|c|c|}
\hline \multirow{2}{*}{ CAL } & \multirow{2}{*}{$\begin{array}{l}\text { TEMPERATURA } \\
\text { DO AMBIENTE } \\
\left({ }^{\circ} \mathrm{C}\right)\end{array}$} & \multirow{2}{*}{$\begin{array}{l}\text { EDS (\% LIMITE DE } \\
\text { RESISTÊNCIA A } \\
\text { TRAÇÃO, } S_{u t} \text { ) }\end{array}$} & \multirow{2}{*}{$\begin{array}{l}\text { CONSTANTE DE POF- } \\
\text { FENBERGER-SWART, K } \\
(\mathbf{M P a} / \mathbf{m m})\end{array}$} & \multicolumn{3}{|c|}{$\begin{array}{c}\text { AMPLITUDE DE TENSÃo } \\
\text { (MPa) }\end{array}$} \\
\hline & & & & \multicolumn{3}{|c|}{$\begin{array}{l}\text { AMPLITUDE DE DESLO- } \\
\text { CAMENTO, } Y_{b}(\mathbf{m m})\end{array}$} \\
\hline \multirow{2}{*}{$\begin{array}{c}900 \\
\mathrm{MCM}\end{array}$} & \multirow{2}{*}{25} & \multirow{2}{*}{20} & \multirow{2}{*}{34,75} & 27,8 & 31 & 34,2 \\
\hline & & & & 0,8 & 0,89 & 0,98 \\
\hline \multirow{2}{*}{$\begin{array}{c}823 \\
\mathrm{MCM}\end{array}$} & \multirow{2}{*}{25} & \multirow{2}{*}{20} & \multirow{2}{*}{32,66} & 26,8 & 28,22 & 31,35 \\
\hline & & & & 0,82 & 0,86 & 0,96 \\
\hline
\end{tabular}

\subsubsection{Ensaios de fadiga por fretting}

Os ensaios de fadiga por fretting foram realizados com uma máquina de ensaios servo hidráulica (MTS), com célula de carga de $5 \mathrm{kN}$, acoplado a um dispositivo pneumático de simulação de fretting que foi idealizado e criado por membros do GFFM, como é possível observar na publicação recente de GARCIA et al. (2019) [21], juntamente com a sua dissertação [22], além dessas publicações é possível encontrar trabalhos que corroboram o funcionamento deste dispositivo [23, 24]. A figura 4 mostra o desenho esquemático desse aparato necessário para realização de ensaios de fadiga por fretting. 


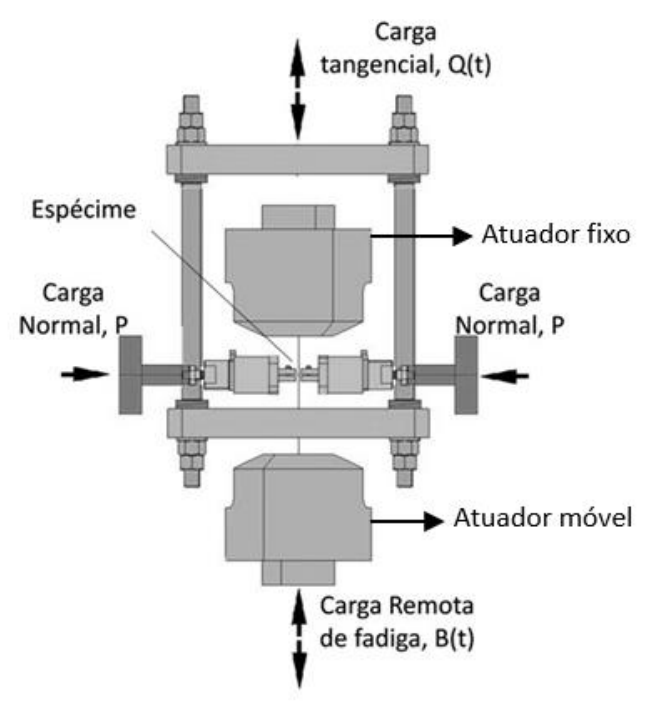

Figura 4: Desenho esquemático do dispositivo de fretting

Esse aparato experimental tem a função de aplicar três cargas independentes ao corpo de prova ensaiado: i) A componente de carga normal a superfície lateral do fio, denominada $\mathrm{P}$, é aplicada com a intenção de induzir um campo de tensões de contato nas regiões de interface da sapata com o corpo de prova (contato fiofio), ii) A carga tangencial $\mathrm{Q}(\mathrm{t})$, que induz o aparecimento de tensões cisalhantes na superfície dos corpos, e iii) A componente $\mathrm{B}(\mathrm{t})$, definida como a carga remota de fadiga, que responsável pela evolução das trincas de fadiga geradas na região de adesão.

Assim como, nos ensaios de fadiga dos materiais e dos condutores, este também possui a presença de força média, com a razão de tensões, $\mathrm{R}=0,1$. Para promover a simulação do fenômeno de fretting existente nos condutores, foi necessário a impressão de carga normal de $750 \mathrm{~N}$ ao fio ensaiado através de uma sapata constituída da mesma liga do fio. Esta sapata foi posicionada a um ângulo de $29^{\circ}$ em relação ao corpo de prova, e para manter o equilíbrio de forças durante o ensaio o outro atuador do dispositivo de fretting utiliza um rolamento para estabelecer o contato na parte posterior do fio. Além disso, a frequência da carga remota de fadiga utilizada nesses ensaios foi de $10 \mathrm{~Hz}$. Este contato é exemplificado pela figura 5. Os corpos de prova utilizados nesta etapa foram retirados de seus respectivos cabos condutores. Logo após foram cortados com comprimento de $220 \mathrm{~mm}$ sem a necessidade de redução de seção, pois, o campo de tensões gerado pelo contato entre o fio e a sapata foi o suficiente para ocorrência da falha. Assim como nos ensaios dos matérias, nesta etapa, foi adotada uma taxa de replicação superior a $20 \%$, portanto, a fim de aumentar a confiabilidade, foram utilizados 5 e 6 corpos de prova, das ligas AA 6201 T81 e AA 1120 H19, respectivamente. 


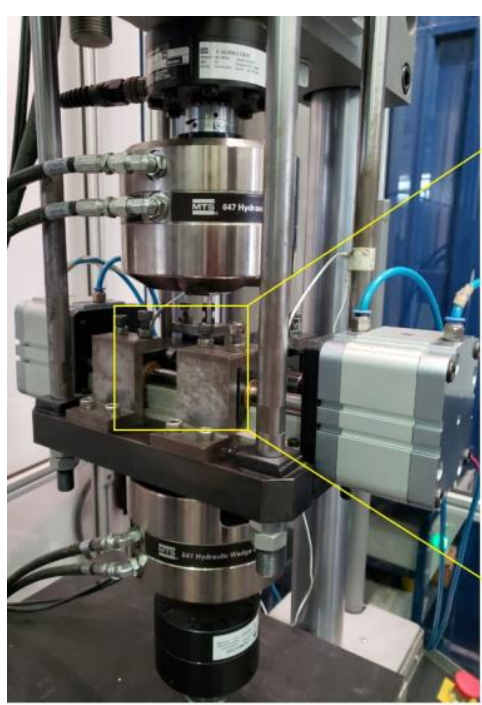

a)

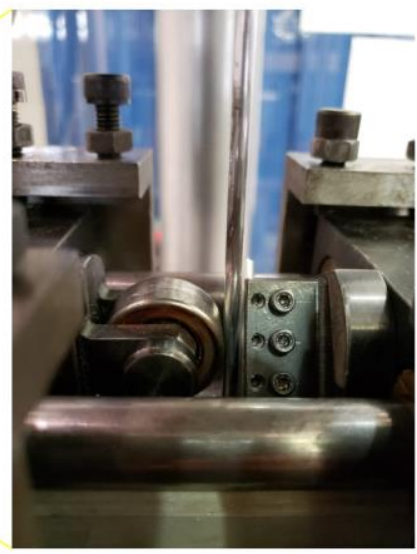

b)

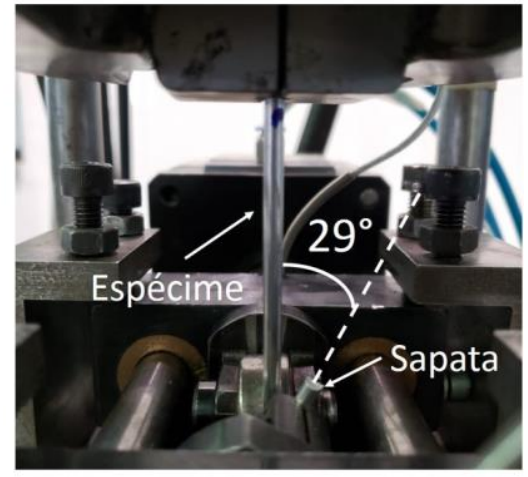

c)

Figura 5: Aparato de esnaio de fadiga por fretting; b) Ampliação da região destacada do contato; c) vista frontal do contato entre o fio e sapata

\section{RESULTADOS E DISCUSSÕES}

\subsection{Resultados da caracterização dos materiais}

Na tabela 4, observa-se que a liga AA 6201 T81 possui propriedades mecânicas estáticas superiores a liga AA 1120 H19.

Tabela 4: Propriedades mecânicas estáticas

\begin{tabular}{cccc}
\hline LIGA & $\begin{array}{c}\text { MOdULO DE ELASTICIDADE, } E, \\
\text { (GPA) }\end{array}$ & $\begin{array}{c}\text { LIMITE DE RESISTENCIA A } \\
\text { TRAÇÃO, } S_{u t}, \text { (MPA) }\end{array}$ & $\begin{array}{c}\text { TENSÃo de ESCOAMENTO, } \\
S_{v} \text {, (MPA) }\end{array}$ \\
\hline AA 6201 T81 & 69 & 330 & 310 \\
\hline AA 1120 H19 & 68,2 & 248,5 & 245 \\
\hline
\end{tabular}

Os resultados dos ensaios dinâmicos são mostrados na figura 6 através de curvas S-N. Nessa figura, os dados experimentais sofreram regressão de potência na forma da equação 1 . 


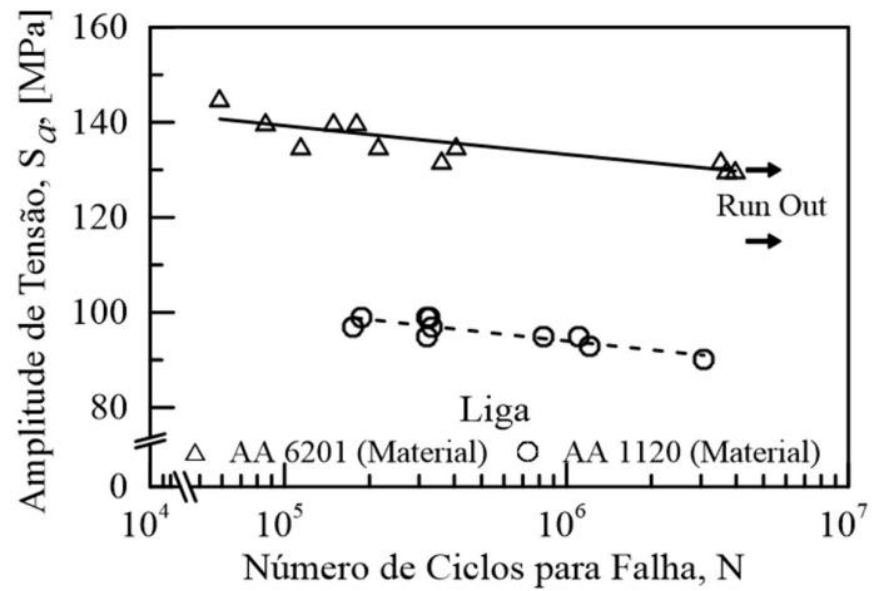

Figura 6: Curvas de fadiga das ligas AA 6201 T81 e AA 1120 H19

Os triângulos e círculos representam os pontos experimentais da liga AA 6201 T81 e AA 1120 H19, respectivamente. Esses dados relacionam a vida do corpo de prova até a sua ruptura em escala logarítmica no eixo horizontal com sua respectiva amplitude de tensão no eixo vertical. A linha contínua representa a curva de fadiga da liga AA 6201 T81, já a linha tracejada representa a curva da liga AA 1120 H19. Ao analisar essas curvas, observa-se que a curva da liga AA 6201 T81 está consideravelmente acima da curva da liga AA 1120 H19 em toda sua extensão. Deste modo pode-se afirmar que a liga AA 6201 T81 possui maior resistência à fadiga em relação a liga AA 1120 H19.

Ao fim dessas análises de caracterização dos materiais, é possível constatar que a liga AA 6201 T81 possui propriedade mecânicas superiores a liga AA 1120 H19, sendo ela, de origem estática ou dinâmica.

\subsection{Resultados dos ensaios de fadiga dos condutores}

Os resultados dos ensaios de fadiga dos condutores 900 MCM e 823 MCM, formados pelas ligas AA 6201 T81 e AA 1120 H19, respectivamente, são mostrados na figura 7. Os dados experimentais desses ensaios também passaram pela mesma regressão de potência como na etapa anterior. Os círculos e triângulos representam os pontos experimentais de cada ensaio de fadiga dos condutores, 823 MCM e 900 MCM, respectivamente.

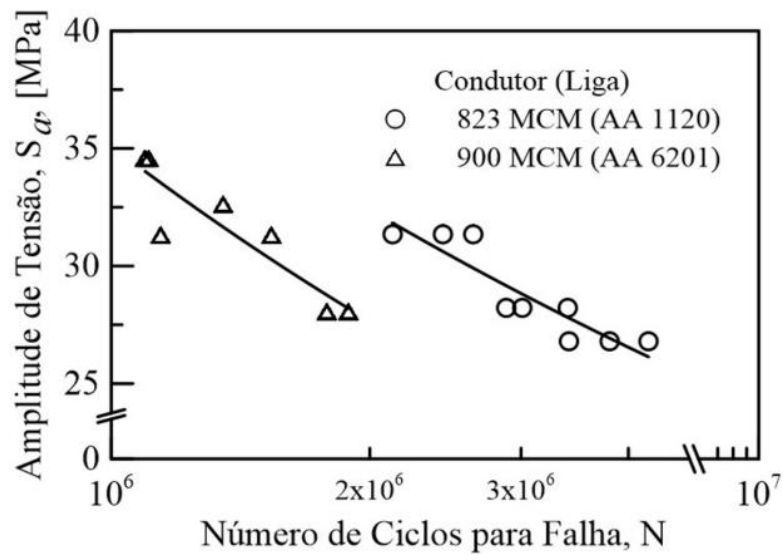

Figura 7: Curvas de fadiga dos condutores 823 MCM e 900 MCM

Ao analisar esses resultados nota-se que a curva representada pelo cabo 832 MCM possui maior resistência à fadiga em relação ao condutor $900 \mathrm{MCM}$. Esse resultado causa certa surpresa quando comparados com os resultados da caracterização dos materiais mostrados na subseção anterior. Pois, sendo o condutor 900 MCM formado pela liga AA 6201 T81, que possui propriedades mecânicas estáticas e de fadiga consideravelmente superiores a liga AA $1120 \mathrm{H} 19$, esperava-se que a resistência desse cabo também fosse superior à do condutor $823 \mathrm{MCM}$. 
Apesar desse resultado ser controverso às expectativas iniciais, ele não é um caso isolado. Em pesquisa recente, o GFFM [8] mostrou resultados bem próximos aos encontrados nesta pesquisa, quando compararam a vida em fadiga dos condutores CAA 397,5 MCM formados pela liga AA 1350 com o CAL 900 MCM. Nesse caso citado, a liga AA 1350 H19 também possui propriedades mecânicas inferiores a liga AA 6201 T81, e mesmo assim o condutor CAA 397,5 obteve maior vida em fadiga em relação ao CAL 900 MCM.

Os resultados desta pesquisa juntamente com o relatado pelo GFFM, apontam indícios de que condutores formados por fios da liga AA 6201 T81 não são tão resistentes à fadiga quando comparados aos cabos formados por fios de ligas da série 1 XXX. Porém, o real motivo para tal ocorrência é difícil de ser encontrado, pois quando o cabo está em operação existem inúmeros problemas mecânicos que são capazes de levar a falha dos condutores. No entanto, neste artigo, adotou-se a ideia de isolar o fenômeno de fadiga por fretting nos fios da liga AA 6201 T81 e AA 1120 H19 em condições controladas, a fim de verificar a influência desse problema mecânico na vida em fadiga dos condutores.

\subsection{Resultados dos ensaios de fadiga por fretting}

Os resultados dos testes de fadiga por fretting também foram apresentados utilizando a metodologia S-N. As curvas resultantes desses testes são apresentadas na figura 8. Para facilitar a análise, esta figura também contém as curvas resultantes dos ensaios de fadiga dos materiais (figura 6), sendo denominadas curvas A e B, onde representam a liga AA 6201 T81 e AA 1120 H19, respectivamente. Nesta figura, os pontos experimentais ilustrados pelos triângulos e círculos vazios representam os resultados dos ensaios de caracterização do comportamento a fadiga das ligas AA 6201 T81 e AA 1120 H19, respectivamente. Contudo, as marcas triangulares e circulares cheias representam os resultados dos ensaios de fadiga por fretting dos fios formados pelas ligas AA 6201 T81 e AA 1120 H19, respectivamente.

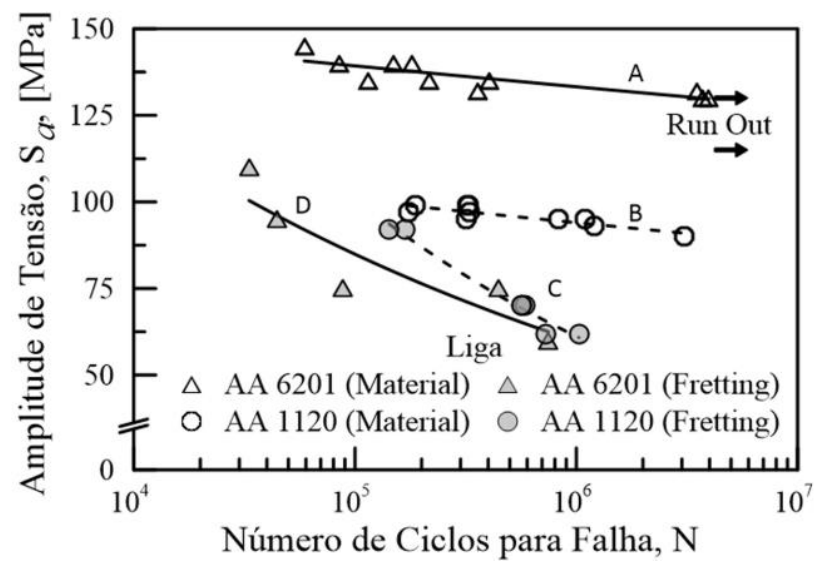

Figura 8: Curvas de fadiga por fretting e curvas de fadiga dos condutores

As curvas C e D representam os resultados de fadiga por fretting das ligas AA 1120 H19 e AA 6201 T81, respectivamente. Ao observar esses resultados experimentais, nota-se que a curva $\mathrm{C}$ possui maior resistência à fadiga em relação a curva $\mathrm{D}$, o que precipitadamente pode não fazer muito sentido, pois, esperava-se que os fios constituídos pela liga mais resistente à fadiga, no caso a AA 6201 T81, também possuísse a maior resistência à fadiga por fretting. Entretanto, assim como nos ensaios de fadiga dos cabos condutores, nesta etapa verificou-se a inversão da resistência à fadiga da liga AA 6201 T81 quando exposta ao contato mecânico.

Por consequência desses resultados, pode-se inferir que a liga AA 6201 T81 possui maior sensibilidade ao fretting em relação a liga AA 1120 H19, para a condição controlada de carga normal de compressão de 750 N. Porém mesmo com essa inferência, a razão para tal ocorrência é mais complexa. Durante a pesquisa foi levantada a hipóteses que a sensibilidade da liga AA 6201 T81 poderia estar relacionada a sua alta dureza, e consequentemente, sua fragilidade, pois, durante o processo de fretting, o contato existente nesse fenômeno estaria agindo como um concentrador de tensões. Deste modo, o comportamento das curvas de fadiga dos fios de liga de alumínio estudas aqui, pode estar relacionado a existência de dois modos básicos da iniciação das trincas de fadiga [25, 26]: modo A, associa-se a condição em que as trincas de fadiga são nucleadas em bandas de deslizamento persistentes, a partir de intrusões e extrusões superficiais e geralmente ocorrem 
quando a tensão máxima é alta e a vida em fadiga é inferior a 106 ciclos; e o Modo B, situação onde as trincas são nucleadas a partir de inclusões e geralmente ocorrem quando a tensão é baixa e a vida de fadiga é superior a 106. Assim, quando se introduz um concentrador de tensão na superfície da componente mecânico durante o contato, a tendência é induzir o processo de iniciação de trinca para essa região específica, que devido ao alto nível de plasticidade local induz a nucleação de trincas em bandas de deslizamento persistentes.

Um aspecto essencial para findar esta análise é a comprovação da ocorrência de fretting durante esses ensaios, e para tal certificação, foi utilizada uma imagem gerada pelo microscópio confocal na região de contato entre o fio e a sapata antes da falha, como é vista na figura 9. Segundo HILLS e NOWELL [27] duas características fundamentais estão presentes em qualquer problema de fretting: primeiro, que na presença de força normal o contato deve estar experimentando tensões de cisalhamento devido a força tangencial transmitida de um corpo ao outro. Em segundo lugar, deve haver algum grau de deslocamento relativo tangencial entre as superfícies de contato, de modo que o deslizamento tome espaço durante a variação cíclica da carga aplicada. Esse regime de deslizamento relativo parcial é expressado pela equação 6.

\section{$Q<f P$}

onde, $Q$ é a força tangencial, $f$ o coeficiente de atrito e $P$ é a força normal.

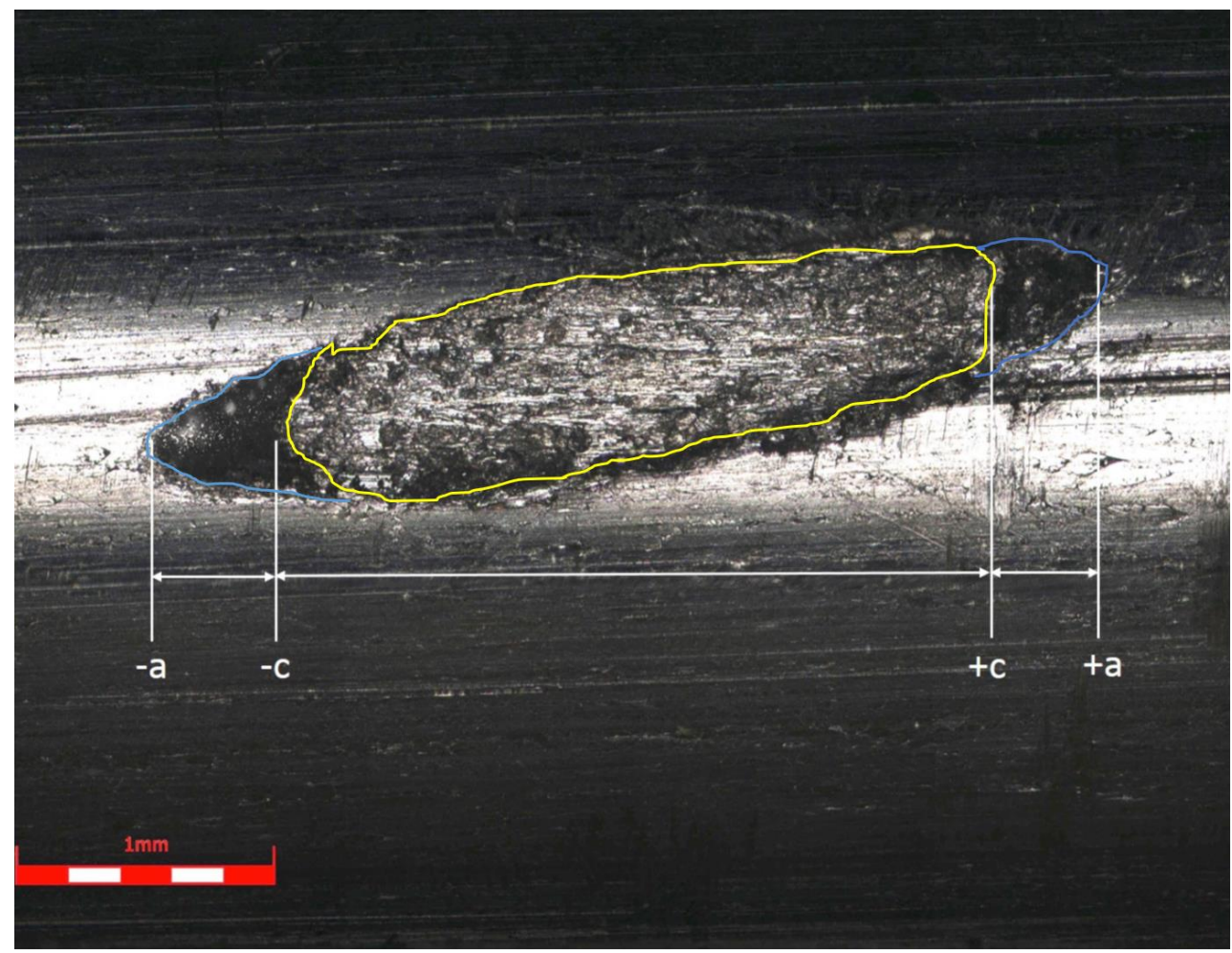

Figura 9: Marca de fretting: zonas de adesão e deslizamento

Quando cilindros elasticamente similares (materiais com o mesmo módulo de elasticidade) estão em contato sob regime de deslizamento parcial, formam-se duas zonas distintas entre si, uma chamada de zona de adesão, onde não há deslocamento relativo, e a outra, chamada de zona de escorregamento, onde ocorre o deslocamento entre as superfícies em contato.

Essas zonas de adesão e de escorregamento são perceptíveis através de imagens adequadas na área de contato, ao investigar a figura 9 , percebe-se a distinção dessas duas regiões. No espaço compreendido entre os intervalos $-c$ e $+c$ é possível observar a zona de adesão, pois está mais clara, menos agredida, mostrando que não houve deslizamento relativo, porém ao olhar os intervalos entre $-a \mathrm{e}-c$ juntamente com $+c \mathrm{e}+a$, nota-se zona de escorregamento, uma área mais escura, sendo um sinal claro que houve escorregamento entre as superfícies. Para facilitar a compreensão dessas zonas, a área de contorno amarelo, representa a zona de adesão, e a área de contorno azul representa as zonas de escorregamento. Com essas evidências apresentadas em relação ao contato entre o fio e a sapata, pode-se inferir que os ensaios de fadiga por fretting, realmente 
ocorreram na presença do fenômeno de fretting.

\section{CONCLUSÕES}

Conclui-se neste artigo que o condutor CAL 900 MCM possui melhor vida em fadiga em relação ao CAL 823 MCM, apesar do primeiro ser formado por fios da liga AA 6201 T81, que é uma liga com maior resistência à fadiga quando comparada a liga AA $1120 \mathrm{H} 19$, formadora dos fios do segundo condutor. A explicação encontrada nesta pesquisa para tal constatação, foi que a liga AA 6201 T81 é significativamente mais sensível ao fretting em relação a liga AA 1120 H19, como foi verificado nos ensaios de fadiga por fretting sob condições controladas.

\section{AGRADECIMENTOS}

A Coordenação de Aperfeiçoamento de Pessoal de Nível Superior (CAPES), a Fundação de Empreendimentos Científicos e Tecnológicos (FINATEC) e também a Transmissoras Brasileiras de Energia (TBE) pelo suporte financeiro para o projeto intitulado Fadiga de Cabos de Alumínio Liga (CAL) 1120 e 6201: Estudo Comparativo, Efeito de Grampos AGS e de Emendas Pré-formadas. Este projeto foi financiado no contexto do Programa de Pesquisa e Desenvolvimento da Agencia Nacional de Energia Elétrica (ANEEL, Brasil).

\section{BIBLIOGRAFIA}

[1] Northeast Group LLC, https://www.prnewswire.com/news-releases/global-electricity-transmission-anddistribution-infrastructure-investment-to-reach-351 bn-per-year-by-2026-300320202.html. Acessado em maio de 2019.

[2] ONS, Plano de Ampliações e Reforços nas Instalações de Transmissão do SIN, PAR Executivo 20192023,"Brasil, 2018.

[3] CIGRE WG B2.30, Engineering Guidlines Relating to Fatigue Endurance Capability of Conductor/Clamp Systems, Techinal Brochures 429: B2 Overhead lines, Oct. 2010.

[4] CIGRE TF B2.11.07, Fatigue Endurance Capability of Conductor / Clamp Systems - Update of Present Knowledge, Technical Brochure 332: B2, Oct. 2007.

[5] IEEE, Guide for aeolian vibration field measurements of overhead conductors,In: IEEE Std 1368, 2007.

[6] FADEL, A.A., ROSA, D., MURÇA, L.B., et al., "Effect of high mean tensile stress on the fretting fatigue life of an Ibis steel reinforced aluminium conductor," International. Journal of Fatigue, v. 42, pp. 24-34, Sep. 2012.

[7] KALOMBO, R.B., "Evaluation of the fatigue resistance of power line conductors functions of the H/w parameter," Tesis D.Sc., ENM/UnB, Brasília, DF, Brasil, 2017.

[8] KALOMBO, R.B., MARTÍNEZ, J.M.G., FERREIRA, J.L.A., et al., "Comparative Fatigue Resistance of Overhead Conductors Made of Aluminium and Aluminium Alloy: Tests and Analysis," Procedia Engineering, v.133, pp. 223-232, 2015.

[9] KALOMBO, R.B., PESTANA, M.S., FERREIRA, J.L.A., et al., "Influence of the catenary parameter (H/w) on the fatigue life of overhead conductors," Tribol. Int., vol. 108, no. November 2016, pp. 141-149, 2017, doi: 10.1016/j.triboint.2016.11.004.

[10] EPRI, Transmission Line Reference Book: Wind Induced Conductor Motion. Palo Alto, CA: Eletrical Power Research Institute, 2009.

[11] EPRI, Transmission line reference book: The Orange Book. Palo Alto, CA: Electric Power Research Institute, 2006.

[12] WITTKOWSKY, B.U., BIRCH, P.R., DOMINGUEZ, J., et al., "An apparatus for quantitative fretting testing," Fatigue \& Fracture of. Engineering. Materials \& Structures, v. 22, n. 4, pp. 307-320, 2003.

[13] FADEL, A.A. "Avaliação do efeito de tracionamento em elevados níveis de EDS sobre a resistência em fadiga do condutor IBIS (CAA 397,5 MCM)", Tesis de D.Sc., ENM/UnB, Brasília, DF, Brasil, 2010.

[14] AZEVEDO, C.R.F., HENRIQUES, A.M.D., PULINO FILHO, et al., "Fretting fatigue in overhead conductors: Rig design and failure analysis of a Grosbeak aluminum cable steel reinforced conductor," Engineering Failure Analysis, v. 16, n. 1, pp. 136-151, Jan. 2009. 
[15] POFFENBERGER, J.C., SWART, R.L., "Differential Displacement and Dynamic Conductor Strain," IEEE Transactions on Power Apparatus and System, v. 84, n. 4, pp. 281-289, 1965.

[16] REINKE,G., "Influência do tratamento térmico nas propriedades mecânicas de fios de alumínio liga usados em cabos condutores submetidos a ensaios de fadiga," ENM/UnB, Brasília, DF, Brasil, 2017

[17] ADRIANO, V.S.R., MARTÍNEZ, J.M.G., FERREIRA, J.L.A., et al.,, "The influence of the fatigue process zone size on fatigue life estimations performed on aluminum wires containing geometric discontinuities using the Theory of Critical Distances," Theoretical and Applied Fracture Mechanics., v.97, no., pp. 265-278, July, 2018, doi: 10.1016/j.tafmec.2018.09.002.

[18] ASTM E739-91, "Standard Practice for Statistical Analysis of Linear or Linearized Stress-Life ( S-N) and Strain-Life ( E-N ) Fatigue Data", pp. 1-7, 2004.

[19] ASTM E466-15, "Standard Practice for Conducting Force Controlled Constant Amplitude Axial Fatigue Tests of Metallic Materials", pp. 1-6, 2015.

[20] CIGRE WG 22.04., "Recommendations for the evaluation of the lifetime of transmission line conductors," Electra, v. 63, pp. 103-145, 1979.

[21] GARCIA, M.A., SILVA, C.R.M., FERREIRA, J.L.A., et al., "Development of a fretting fatigue device to test aluminum wires," in 25th ABCM International Congress of Mechanical Engineering, p. 10, doi: 10.26678/ABCM.COBEM2019.COB2019-0162.

[22] GARCIA, M.A., "Concepção, fabricação e avaliação de um dispositivo de fadiga por fretting em fios de alumínio de cabos condutores," ENM/UnB, Brasília, DF, Brasil, 2019.

[23] ROCHA, P.H.C., DÍAZ, J.I.M., SILVA, C.R.M., et al., "Fatigue of two contacting wires of the ACSR Ibis 397.5 MCM conductor: Experiments and life prediction," International Journal of Fatigue, v. 127, no., pp. 25-35, May, 2019, doi: 10.1016/j.ijfatigue.2019.05.033.

[24] DÍAZ, J.I.M., "Avaliação do efeito de fadiga por fretting sobre a resistência do fio no cabo condutor Ibis (caa 397,5mcm).” ENM/UnB, Brasília, DF, Brasil, 2019.

[25] MUGHRABI, H., "Specific features and mechanisms of fatigue in the ultrahigh-cycle regime," International Journal of Fatigue, v. 28, no. 11, pp. 1501-1508, 2006, doi: 10.1016/j.ijfatigue.2005.05.018.

[26] MARINES, I., BIN, X., BATHIAS, C., "An understanding of very high cycle fatigue of metals," Interational Journal of Fatigue, v. 25, no. 9-11, pp. 1101-1107, 2003, doi: 10.1016/S0142-1123(03)001476.

[27] HILLS, D.A., NOWELL, D., Mechanics of Fretting Fatigue, 1st ed. Dordrecht/Boston/London: Kluwer Academic Publishers, 1994.

\section{ORCID}

Erick Rocha Vieira

Jorge Luiz de Almeida Ferreira

José Alexander Araújo

Remy Badibanga Kalombo

Cosme Roberto Moreira da Silva
https://orcid.org/0000-0001-8087-328X

https://orcid.org/0000-0002-1816-9707

https://orcid.org/0000-0002-0025-759X

https://orcid.org/0000-0003-2887-9670

https://orcid.org/0000-0001-6301-6446 